\title{
Celiac disease and alcohol use disorders: Increased length of hospital stay, overexpenditures and attributable mortality
}

\author{
Miguel Gili1,2, Luis Béjar², Gloria Ramírez ${ }^{1,2}$, Julio López ${ }^{1,2}$, Juan Luis Cabanillas ${ }^{2,3}$ and Brett Sharp ${ }^{4}$ \\ ${ }^{I}$ Unit of Preventive Medicine, Surveillance and Health Promotion. Hospital Universitario Virgen Macarena. Sevilla, \\ Spain. ${ }^{2}$ Department of Preventive Medicine and Public Health. School of Medicine. Universidad de Sevilla. Sevilla, \\ Spain. ${ }^{3}$ Consejería de Salud de la Junta de Andalucía. Sevilla, Spain. ${ }^{4}$ Clinical Unit of Diagnostic Image. Hospital \\ Universitario Virgen de Rocío. Sevilla, Spain
}

\begin{abstract}
Background and objectives: alcohol use disorders are associated with a greater incidence of certain comorbidities in patients with celiac disease. Currently there is no available information about the impact that these disorders may have on length of hospital stays, overexpenditures during hospital stays, and excess mortality in these patients.

Methods: a case-control study was conducted with a selection of patients 18 years and older hospitalized during 2008-2010 in 87 hospitals in Spain. Estimations of excess length of stays, costs, and attributable mortality were calculated using a multivariate analysis of covariance, which included age, gender, hospital group, alcohol use disorders, tobacco related disease and 30 other comorbidities.

Results: patients who had both celiac disease and alcohol use disorders had an increased length of hospital stay, an average of 3.1 days longer in women, and 1.7 days longer in men. Excess costs per stay ranged from 838.7 euros in female patients, to 389.1 euros in male patients. Excess attributable mortality was $15.1 \%$ in women, $12.2 \%$ in men.

Conclusions: apart from a gluten-free diet and other medical measures, the prevention of alcohol abuse is indicated in these patients. Patients hospitalized who present these disorders should receive specialized attention after leaving the hospital. Early detection and treatment should be used to prevent the appearance of organic lesions and should not be solely focused on male patients.
\end{abstract}

Key words: Celiac disease. Alcohol. Hospital stay. Costs. Mortality.

\section{INTRODUCTION}

Celiac disease has a variable prevalence, with an average that varies between $0.5 \%$ and $1 \%$ according to studies performed in Europe and North America (1-6). In Europe,

Received: 19-07-2013

Accepted: 25-09-2013

Correspondence: Miguel Gili. Unit of Preventive Medicine, Surveillance and Health Promotion. Hospital Universitario Virgen Macarena. Avda. del Dr. Fedriani, s/n. 41070 Sevilla. Spain

e-mail:mgili@us.es different countries register great discrepancies $(0.3 \%$ in Germany versus $2.4 \%$ in Finland in people between 30 and 64 years of age), for reasons still unclear (6). Prevalence of celiac disease is between 1.5 and 2 times greater in women than in men (7-9), and some studies suggest that it is increasing (8-12).

Celiacs usually present with problems related to malabsorption of iron and folic acid, made worse by the consumption of large quantities of alcohol (13). Among the complications related to celiac disease, many are worsened if the patient consumes in excess alcoholic beverages, for example, peripheral neuropathy $(14,15)$ and epilepsy $(16)$. Abnormal levels of aminotransferases (17) can be found in patients with celiac disease, as well as an association with several autoimmune chronic liver diseases such as primary biliary cirrhosis $(18,19)$. There is also an increased risk of autoimmune cardiomyopathy $(20,21)$.

When these patients have alcohol use disorders, such problems can be seriously aggravated. In fact, any pathology associated with celiac disease, such as metabolic alterations (diabetes type I), neoplasia (tumors of the small intestine and lymphomas), among others, can favor the apparition of complications that worsen the vital prognosis of these patients and increase the length of their hospital stays (22).

For these reasons, the objective of this study is to evaluate the impact that alcohol use disorders have among hospitalized patients already diagnosed with celiac disease, through the analysis of factors such as increased length of hospital stay, excess costs and attributable mortality. In order to do so, this problem was studied in hospitalized patients aged 18 and older in a sample of 87 Spanish hospitals during the period of 2008-2010, while controlling

Gili M, Béjar L, Ramírez G, López J, Cabanillas JL, Sharp B. Celiac disease and alcohol use disorders: Increased length of hospital stay, overexpenditures and attributable mortality. Rev Esp Enferm Dig 2013;105:537-543. 
confounding and interacting factors such as age, gender, type of hospital, tobacco related disorders and a considerable number of comorbidities.

\section{METHODS}

\section{Participants}

Data for hospital admissions and readmissions were captured in the administrative minimal basic data set (MBDS) of 87 Spanish hospitals during the period of 2008-2010. The study was designed as a case-control study. Sample size for this study, which analyzes problems related to the consumption of alcohol, tobacco, and other substances of abuse, was calculated admitting a $5 \%$ alpha error rate, a two tailed test, had a power of $90 \%$, a relationship of non-case:case of at least 4:1, and the least favorable proportion of cases in which the cases are exposed and the non-cases are not exposed according to available scientific evidence was used. In order for the sample to be representative both on a national and regional scale, and keeping in mind the stratification of hospitals according to their size and complexity in the classification set forth by the Ministry of Health, sampling was done in various stages in which 87 Spanish hospitals were selected from all of the autonomous regions of Spain.

From written or digitalized information that was provided by the hospital physician who signs the clinical record, each patient's diagnosis, external causes and procedures were codified according to International Classification of Diseases, $9^{\text {th }}$ review (ICD-9-CM) codes. Codification and data entry in the electronic database are performed by dedicated administrative personnel who have completed in-depth training on medical data registration. This administrative database has demographic data, admission and discharge dates, type of admission and type of discharge, diagnostic codes for principal cause and secondary diagnoses, external causes and procedures using the ICD-9CM. Also included in these databases are diagnosis-related groups (DRG) and a classification of each hospital into five categories based on size and complexity of services, varying from those with a smaller size and lesser complexity to larger hospitals that offer a broader range of services (23). Analysis was restricted to patients who at the moment of hospital discharge were 18 and older.

\section{Variables}

Cases of celiac disease were defined as those which fit the codes specified in the ICD-9-CM as a primary or secondary diagnosis (code 579.0). Alcohol related disorders were defined as alcohol dependence (303.00-303.93), alcohol abuse (305.00-305.03), alcohol-induced mental disorders (291.0-291.9), alcoholic polyneuropathy (357.5), alcoholic cardiomyopathy (425.5), alcoholic gastritis (535.30-535.31), alcoholic liver disorders (571.0-571.3), excessive blood alcohol level (790.3), alcohol toxicity and alcohol poisoning (980.0-980.9 and E860.0-E860.9). The ICD-9-CM code for disorders associated with tobacco dependence was used (305.1).

Age was measured in years. The following comorbidities were identified: Obesity, uncomplicated hypertension, complicated hypertension, cardiac arrhythmias, pulmonary circulation disorders, valvular disease, deficiency anemia, blood loss anemia, fluid and electrolyte disorders, weight loss, hypothyroidism, coagulopathy, myocardial infarction, congestive heart failure, peripheral vascular disorders, cerebrovascular disease, dementia, chronic pulmonary disease, rheumatic disease, peptic ulcer disease, mild liver disease, diabetes without chronic complications, diabetes with chronic complications, hemiplegia or paraplegia, renal disease, moderate or severe liver disease, cancer, leukemia or lymphoma, metastatic cancer, AIDS and depression. We used the ICD-9-CM codes proposed for these comorbidities by Quan et al. (24). Charlson Comorbidity Index was also calculated for every patient (25).

\section{Data analysis}

The primary outcome of interest was to determine the length of hospital stays, costs and mortality in patients with celiac disease with or without alcohol use disorders. Costs were calculated using specific hospital spending data organized according to DRG and stratified by hospital group, using estimates published by the Ministry of Health during 2008, 2009, and 2010 (23).

Univariate analysis was used to examine the association between celiac disease and alcohol use disorders, as well as tobacco related disorders, age, gender, and comorbidities. In order to compare continuous variables a Student's t-test or a parametric equivalent was used. For qualitative variables a Chi-squared test was used.

Multivariate analysis of covariance was performed to determine the effect of alcohol use disorders on hospital length of stay (in days), costs and mortality in patients with celiac disease. Data were adjusted for age, gender, tobacco related disorders, group of hospitals and severity of disease using the 30 comorbidities before mentioned. Analysis was performed in STATA version MP 12.1.

\section{RESULTS}

\section{Patient characteristics}

Patient characteristics with celiac disease and without celiac disease are detailed in table I. A total of 5,475,315 patients were identified, of which 3,327 were admitted with celiac disease, while 344,429 had alcohol use dis- 
orders. The prevalence of alcohol use disorders was lower among patients with celiac disease $(4.9 \%)$ than in the rest of patients $(6.3 \%)$, but with extraordinary differences according to age group and gender, as seen in figure 1 . Male celiac patients between the ages of 55-64 years had the highest prevalence of alcohol use disorders $(19.3 \%)$ while in female celiac patients it was highest in those of $35-44$ years of age $(3.6 \%)$. The prevalence of tobacco related disorders was lower in patients with celiac disease than in the rest of patients (17.8\% versus $20.3 \%$ ).

Patients diagnosed with celiac disease were significantly younger (average age: 48.7 years versus 57.6 ) and mostly female (70.2\% of the cases).

Patients with celiac disease had fewer comorbidities than the rest of the patients, as shown by the average Charlson Comorbidity Index (0.89) compared to the rest of hospitalized patients (1.19). However, there are a series of comorbidities classically associated with celiac disease, which in this study also demonstrated higher prevalence: Anemia, electrolyte disorders, weight-loss, hypothyroidism, autoimmune rheumatic diseases, depression, liver disorders and associated disorders such as coagulopathies.

\section{Prolongation of hospital stays, excess costs and attributable mortality}

A differential analysis was performed for female and male patients. In-hospital mortality in patients with celiac disease was greater in males than in females (3.9\% versus $2.2 \%, \mathrm{p}=0.0046)$. In-hospital mortality in males with celiac disease and alcohol use disorders was inferior than in female patients with celiac disease and alcohol use disorders (3.4\% versus $4.3 \%$ ), though the difference was not found to be statistically significant ( $\mathrm{p}=0.8042)$.

Adjusted estimates of excess length of hospital stays, costs per stay and attributable mortality due to alcohol use disorders, which resulted from multivariate analysis of covariance are detailed in table II. One can see that the prolongation of hospital stays (in days) in female patients with celiac disease and alcohol use disorders in comparison to females with celiac disease and without alcohol use disorders is almost double that of male patients (3.1 days versus 1.7 days). Due to this difference, the excess costs per stay in euros in female patients with celiac disease and alcohol use disorders compared to female celiac disease patients without these disorders more than doubled that of male patients (838.7 euros versus 389.1 euros).

In contrast to the greater in-hospital mortality in male patients with celiac disease, the excess mortality attributable to alcohol use disorders among female patients with celiac disease in comparison with those without alcohol use disorders was greater than in male patients $(15.1 \%$ versus $12.2 \%$ ).

\section{DISCUSSION}

This study shows that patients with celiac disease and alcohol use disorders have increased lengths of hospital stays, incur greater costs during their stay, and have higher mortality rates. These effects are increased in women.

The relationship between alcohol use disorders and celiac disease is complex and influenced by many factors. As mentioned earlier, alcohol abuse and disorders associated with excess consumption worsen the evolution and prognosis of celiac disease and associated autoimmune disorders.

Not only that, there are other relationships that exist involving the immune system. In the gut, there are abundant enzymes that metabolize ethanol into acetaldehyde, a very reactive compound that is thought to have an important role in the pathogenesis of various alcohol use disorders of the digestive tract (26-28). Some studies show that patients with increased consumption of ethanol may produce IgA-mediated immune responses to proteins containing epitopes modified by the action of acetaldehyde (2931 ). It has also been shown that the reactions catalyzed by transglutaminases are involved in the pathogenesis of celiac disease (32). In a study in which IgA antibodies active against tissue transglutaminases and proteins modified by acetaldehyde were measured, levels of both these antibodies were significantly higher in patients with alcoholic liver disease and in heavy drinkers without liver disease, showing a direct correlation with levels of biomarkers of ethanol consumption, pro-inflammatory cytokines, and markers of fibrogenesis (33). These findings support the hypothesis that elevated and persistent consumption of alcohol can generate other sources of antigen production and abnormal activation of tissue transglutaminases. Finally, other studies have found that increased levels of antibodies active against transglutaminases may be associated with a greater risk of mortality in the general population, which increases the potential impact that alcohol abuse and its interactions have on mortality in patients with celiac disease (34).

Our study has several limitations. The data used were only those found in the MBDS and were not complemented with additional data from patients. We used clinical definitions of celiac disease, alcohol and tobacco related disorders and comorbidities as assigned by physicians and entered by codifiers. Another limitation is potential underreporting if all of the information required to complete such codes is not available in the clinical record, or due to variations in the codifiers interpretation of data. The ICD-9-CM does not include codes specific to the motive for hospitalizing patients, and in some cases, this would make an interesting variable to study.

Databases such as the MBDS also have clear advantages (35). The data collected are usually completed in most hospital admissions and, as they include virtually all cases, provide reasonably accurate estimates of the incidence, prevalence, comorbidity, and mortality of diseases treated 
Table I. Characteristics of patients with and without celiac disease

\begin{tabular}{|c|c|c|c|c|c|}
\hline \multirow[t]{2}{*}{ Variables } & \multicolumn{2}{|c|}{$\begin{array}{l}\text { With celiac disease } \\
\quad(n=3,327)\end{array}$} & \multicolumn{2}{|c|}{$\begin{array}{l}\text { Without celiac disease } \\
\quad(n=5,471,988)\end{array}$} & \multirow[t]{2}{*}{$p$} \\
\hline & $n$ & $\%$ & $n$ & $\%$ & \\
\hline Alcohol use disorders (all) ${ }^{1}$ & 163 & 4.9 & 344,266 & 6.3 & 0.0009 \\
\hline Alcohol abuse & 89 & 2.7 & 171,091 & 3.1 & 0.1346 \\
\hline Alcohol Induced mental disorders & 6 & 0.2 & 19,320 & 0.4 & 0.0931 \\
\hline Alcoholic liver disorder & 41 & 1.2 & 94,042 & 1.7 & 0.0310 \\
\hline Alcohol toxicity and alcohol poisoning & 1 & 0.03 & 2,303 & 0.04 & 0.7352 \\
\hline Tobacco use disorders & 592 & 17.8 & $1,109,951$ & 20.3 & 0.0004 \\
\hline Average age in years $(\mathrm{Cl} 95 \%)^{2}$ & \multicolumn{2}{|c|}{$48.7(48.1-49.4)$} & \multicolumn{2}{|c|}{$57.6(57.6-57.6)$} & $<0.0001$ \\
\hline \multicolumn{6}{|l|}{ Gender } \\
\hline Group 1 & 32 & 1.0 & 64,456 & 1.2 & 0.2481 \\
\hline Group 2 & 177 & 5.3 & 349,318 & 6.4 & 0.0121 \\
\hline Group 3 & 1,197 & 36.0 & $1,911,738$ & 34.9 & 0.2078 \\
\hline Group 4 & 879 & 26.4 & $1,482,647$ & 27.1 & 0.3812 \\
\hline Group 5 & 1,042 & 31.3 & $1,663,829$ & 30.4 & 0.2524 \\
\hline \multicolumn{6}{|l|}{ Comorbidities } \\
\hline Obesity & 71 & 2.1 & 246,383 & 4.5 & $<0.0001$ \\
\hline Uncomplicated hypertension & 506 & 15.2 & $1,419,229$ & 25.9 & $<0.0001$ \\
\hline Complicated hypertension & 94 & 2.8 & 313,936 & 5.7 & $<0.0001$ \\
\hline Cardiac arrhythmias & 254 & 7.6 & 757,093 & 13.8 & $<0.0001$ \\
\hline Pulmonary circulation disorders & 56 & 1.7 & 136,749 & 2.5 & 0.0026 \\
\hline Valvular disease & 147 & 4.4 & 283,768 & 5.2 & 0.0460 \\
\hline Peripheral vascular disorders & 2 & 0.06 & 4,894 & 0.09 & 0.5716 \\
\hline Cerebro-vascular disease & 84 & 2.5 & 275,373 & 5.0 & $<0.0001$ \\
\hline Dementia & 15 & 0.5 & 118,954 & 2.2 & $<0.0001$ \\
\hline Chronic pulmonary disease & 305 & 9.2 & 654,990 & 12.0 & $<0.0001$ \\
\hline Rheumatic disease & 61 & 1.8 & 63,046 & 1.2 & 0.0002 \\
\hline Peptic ulcer disease & 43 & 1.3 & 47,376 & 0.9 & 0.0079 \\
\hline Mild liver disease & 199 & 6.0 & 146,566 & 2.7 & $<0.0001$ \\
\hline Diabetes without chronic complications & 263 & 7.9 & 693,912 & 12.7 & $<0.0001$ \\
\hline Diabetes with chronic complications & 85 & 2.6 & 152,138 & 2.8 & 0.4291 \\
\hline Hemiplegia or paraplegia & 14 & 0.4 & 48,081 & 0.9 & 0.0047 \\
\hline Renal disease & 51 & 1.5 & 106,655 & 1.9 & 0.0825 \\
\hline Moderate or severe liver disease & 104 & 3.1 & 77,207 & 1.4 & $<0.0001$ \\
\hline Cancer, leukemia o lymphoma & 321 & 9.6 & 701,836 & 12.8 & $<0.0001$ \\
\hline Metastatic cancer & 100 & 3.0 & 257,174 & 4.7 & $<0.0001$ \\
\hline AIDS & 3 & 0.09 & 32,715 & 0.6 & 0.0001 \\
\hline Depression & 226 & 6.8 & 216,527 & 4.0 & $<0.0001$ \\
\hline Average Charlson Index (Cl $95 \%)$ & \multicolumn{2}{|c|}{$0.89(0.84-0.95)$} & \multicolumn{2}{|c|}{$1.19(1.19-1.19)$} & $<0.0001$ \\
\hline
\end{tabular}




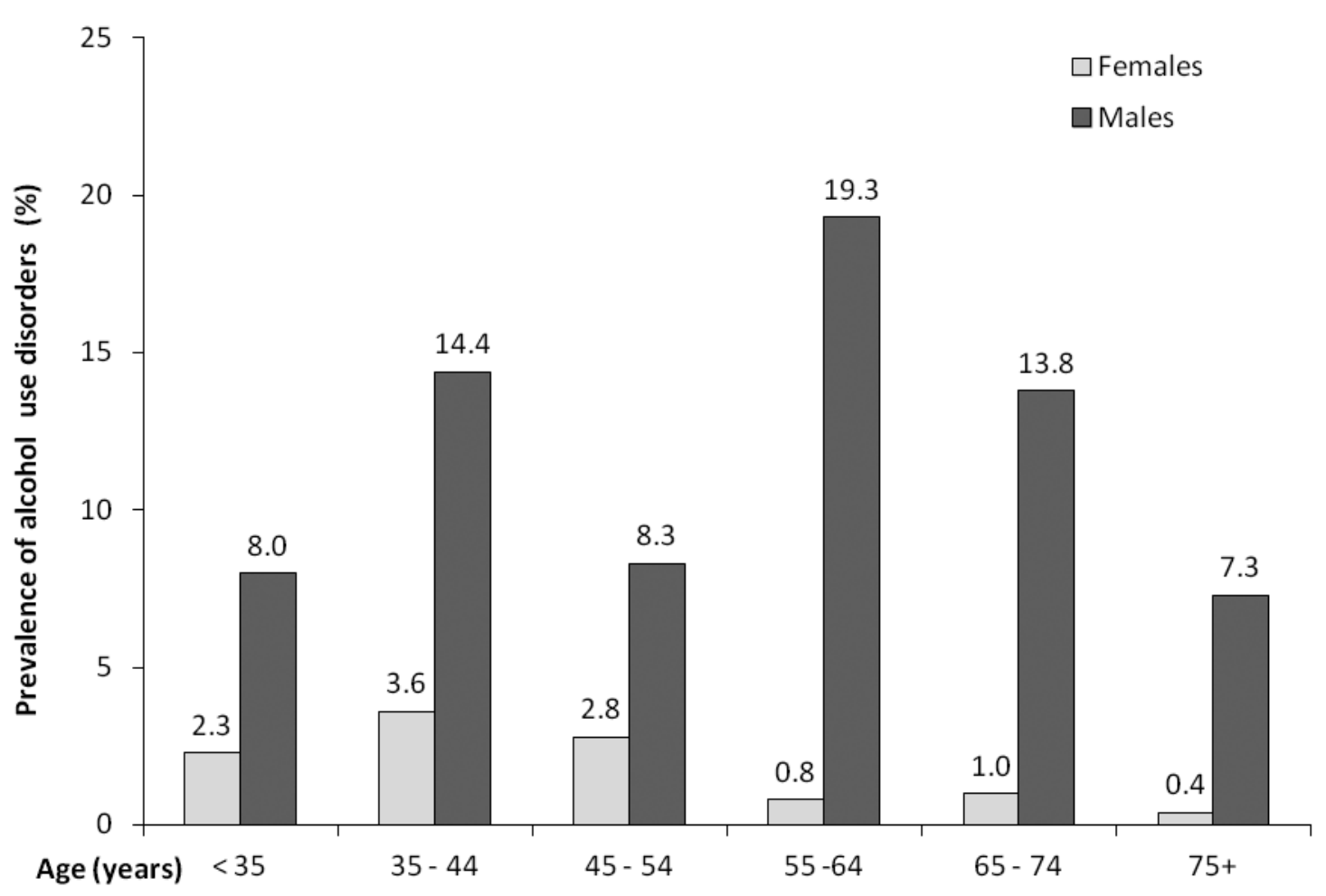

Fig. 1. Distribution of the prevalence of alcohol use disorders according to age and gender among hospitalized patients with celiac disease.

Table II. Prolongation of hospital stays, excess costs per stay and mortality attributable to alcohol use disorders in hospitalized patients with celiac disease

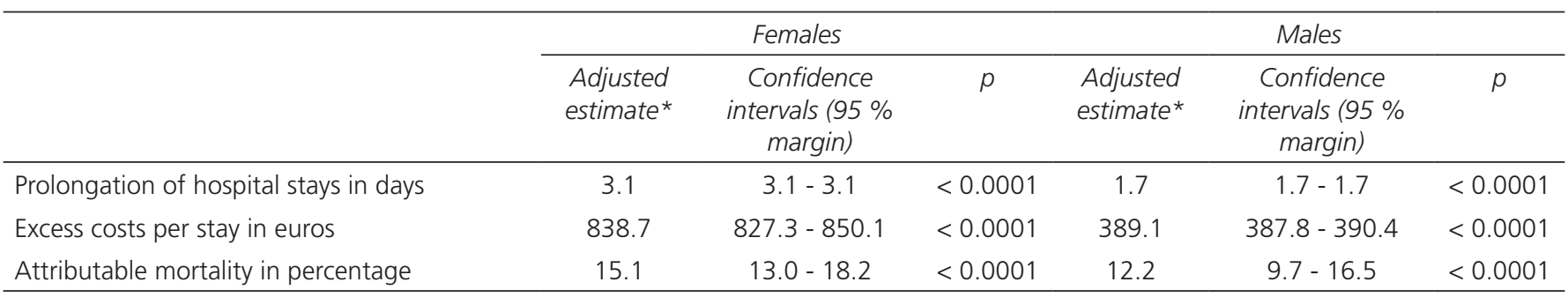

*Calculated with a multivariate analysis of covariance that includes for each gender: Age, hospital group, tobacco use disorders, and the 30 comorbidities described in the text.

in the hospital setting (36). The data can be analyzed retrospectively, unlike other designs that require prospective data collection, and data from long periods or from large numbers of patients can be gathered quickly and easily. Since the data are collected systematically, costs are considerably reduced. In studies using these databases, there may be less selection bias due to the refusal of patients or their legal representatives to sign consent forms, which would allow the patient to participate in the study.

Because of our large sample size and diversity of hospitals, our data are generalizable and not limited to patients admitted to a small number of centers. The availability of costs of hospitalization for each DRG, stratified by hospital group and specific to each year, facilitates the calculation of excess costs due to prolonged stays attributed to alcohol use disorders. As far as we know, this is the first study of it's kind in which excess length of stays, costs, and mortality attributed to alcohol use disorders among patients with celiac disease have been examined.

There are various possible explanations for the greater impact seen in women rather than men. Mortality was discretely increased in women with celiac disease and alcohol use disorders ( $4.3 \%$ versus $3.4 \%$ ), despite the fact that the average age of men was greater (Fig. 1), and could be explained by cases among women included that had worse prognosis and increased length of stays and costs (massive cerebral hemorrhage and irreversible hepatic coma). The results for attributable mortality among women have ample 
margins of confidence (13.0-18.2) and overlap in part with those of men (7.9-16.5), for this reason these differences should be analyzed with caution. On the other hand, the relative percentage of impact is greater in women than in men, because among women in general, there are not as many competing variables of exposition that prolonged hospital stays and increased costs (tobacco dependence, drug use, diet and many other dangerous behaviors more prevalent in males). In the analysis, the increased relative weight of alcohol use disorders controlling the rest of these problems could have influenced the estimations made in the multivariable models. We have no information as to the social class and marital status of these patients, nor the distribution according to gender, factors that could also have influenced the length of hospital stays. Finally, we cannot rule out that complications not reflected in the MBDS may have influenced these outcomes in female patients.

Some studies demonstrate that the prevalence of autoimmune diseases in patients with celiac disease is intimately linked with the duration of the exposure to gluten (37). It has been demonstrated that the antibodies responsible for autoimmunity in diabetes mellitus I and autoimmune thyroiditis can disappear when children with celiac disease consume a gluten-free diet (38), and the scientific evidence indicates that various autoimmune diseases can improve if these patients are prescribed a gluten-free diet $(39,40)$. These authors suggest that many autoimmune diseases could be prevented through the early diagnosis and treatment of celiac disease and, even in patients with an established disorder, a gluten-free diet could decrease symptoms, improve prognosis, and decrease the risk of hospital readmissions. It is plausible that adherence to a gluten-free diet may be worse among patients with alcohol use disorders, who often do not comply with medical advice. According to the results of this study, it is safe to suggest that the diagnosis and treatment of alcohol abuse should be, together with a gluten-free diet and other treatments, one of the principal therapeutic objectives after these patients are discharged. A brief intervention detailing the risks of alcohol and tobacco consumption, and if necessary, referral to a treatment center have been demonstrated to be effective measures (41), and can prevent complications and readmissions in patients with celiac disease. These problems should also be carefully watched for in women, with a focus on early detection and prevention, as there is a greater relative impact than in men. It would also be very interesting to study the influence that alcohol abuse and dependence have in patients in which irreversible organic lesions have not yet appeared. Reducing the number of hospital admissions and readmissions attributed to alcohol use disorders in these patients would help to reduce costs and increase treatment availability in hospitals. Early intervention and prevention of readmissions of patients suffering from pathology associated with celiac disease and alcohol use disorders can also help reduce the burden on patients and family members.

\section{ACKNOWLEDGEMENTS}

This study was funded by the Delegación del Gobierno, Plan Nacional Sobre Drogas. Ministerio de Sanidad, Servicios Sociales e Igualdad (Ayuda N ${ }^{\circ}$ 2009I017, Proyecto G41825811).

\section{REFERENCES}

1. West J, Logan RF, Hill PG, Lloyd A, Lewis S, Hubbard R, et al. Seroprevalence, correlates, and characteristics of undetected coeliac disease in England. Gut 2003;52:960-5.

2. Fasano A, Berti I, Gerrarduzzi T, Not T, Colletti RB, Drago S, et al. Prevalence of celiac disease in at-risk and not-at-risk groups in the United States: A large multicenter study. Arch Intern Med 2003;163:286-92.

3. Green PH, Jabri B. Coeliac disease. Lancet 2003;362:383-91.

4. Evans KE, Sanders DS. Celiac Disease. Gastroenterol Clin N Am 2012;41:639-50.

5. Biagi F, Klersy C, Balducci D, Corazza GR. Are we not over-estimating the prevalence of celiac disease in the general population? Ann Med 2010;42:557-61.

6. Mustalahti K, Catassi C, Reunanen A, Fabiani E, Heier M, McMillan S, et al. The prevalence of celiac disease in Europe: Results of a centralized, international mass screening project. Ann Med 2010;42:587-95.

7. Fasano A, Catassi C. Celiac disease. N Engl J Med 2012;367:2419-26.

8. Rodrigo L, Pérez I. Enfermedad celiaca el adulto: un problema de salud frecuente, importante y de distribución mundial. Rev Esp Enferm Dig 2010;102:461-5.

9. Fernández A, González L, de la Fuente J. Enfermedad celiaca: formas de presentación en el adulto. Rev Esp Enferm Dig 2010;102:466-71.

10. Catassi C, Kryszak D, Bhatti B, Sturgeon C, Helzlsouer K, Clipp SL, et al. Natural history of celiac disease autoimmunity in a USA cohort followed since 1974. Ann Med 2010;42:530-8.

11. Lohi S, Mustalahti K, Kaukinen K, Laurila K, Collin P, Rissanen H, et al. Increasing prevalence of coeliac disease over time. Aliment Pharmacol Ther 2007;26:1217-25.

12. Rubio-Tapia A, Kyle RA, Kaplan EL, Johnson DR, Page W, Erdtmann $\mathrm{F}$, et al. Increased prevalence and mortality in undiagnosed celiac disease. Gastroenterology 2009;137:88-93

13. Rehm J, Room R, Graham K, Monteiro M, Gmel G, Sempos CT. The relationship of average volume of alcohol consumption and patterns of drinking to burden of disease -An overview. Addiction 2003;98:1209-28.

14. Alaedini A, Green PH, Sander HW, Hays AP, Gamboa ET, Fasano A, et al. Ganglioside reactive antibodies in the neuropathy associated with celiac disease. J Neuroimmunol 2002;127:145-8.

15. Chin RL, Sander HW, Brannagan TH, Green PH, Hays AP, Alaedini A, et al. Celiac neuropathy. Neurology 2003;60:1581-5.

16. Molteni N, Bardella MT, Baldassarri AR, Bianchi PA. Celiac disease associated with epilepsy and intracranial calcifications: Report of two patients. Am J Gastroenterol 1988;83:992-4.

17. Bardella MT, Fraquelli M, Quatrini M, Molteni N, Bianchi P, Conte D. Prevalence of hypertransaminasemia in adult celiac patients and effect of gluten-free diet. Hepatology 1995;22:833-86.

18. Dickey W, McMillan SA. Co-screening for primary biliary cirrhosis and coeliac disease. Association between primary biliary cirrhosis and coeliac disease. Gut 1998;43:300.

19. Dickey W, McMillan SA, Callender ME. High prevalence of celiac sprue among patients with primary biliary cirrhosis. J Clin Gastroenterol 1997;25:328-9.

20. Fonager K, Sorensen HT, Norgard B, Thulstrup AM. Cardiomyopathy in Danish patients with coeliac disease. Lancet 1999;354:1561.

21. Frustaci A, Cuoco L, Chimenti C, Pieroni M, Fioravanti G, Gentiloni $\mathrm{N}$, et al. Celiac disease associated with autoimmune myocarditis. Circulation 2002; 105:2611-8.

22. Room R, Babor T, Rehm J. Alcohol and public health. Lancet 2005;365:519-30.

23. Ministerio de Sanidad, Servicios Sociales e Igualdad. Registro de Altas de los Hospitales Generales del Sistema Nacional de Salud. CMBD. 
Norma Estatal. 2011. Disponible en: http://www.msc.es/estadEstudios/ estadisticas/cmbd.htm .

24. Quan H, Sundararajan V, Halfon P, Fong A, Burnand B, Luthi JC, et al. Coding algorithms for defining comorbidities in ICD-9-CM and ICD-10 administrative data. Med Care 2005;43:1130-9.

25. Charlson ME, Pompei P, Ales KL, McKenzie CR. A new method of classifying prognostic comorbidity in longitudinal studies: Development and validation. J Chronic Dis 1987;40:373-83.

26. Salaspuro MP. Acetaldehyde, microbes, and cancer of the digestive tract. Crit Rev Clin Lab Sci 2003;40:183-208.

27. Seitz HK, Gartner U, Egerer G, Simanowski UA. Ethanol metabolism in the gastrointestinal tract and its possible consequences. Alcohol Alcohol Suppl 1994;2:157-62.

28. Visapää JP, Jokelainen K, Nosova T, Salaspuro M. Inhibition of intracolonic acetaldehyde production and alcoholic fermentation in rats by ciprofloxacin. Alcohol Clin Exp Res 1998;22:1161-4.

29. Latvala J, Hietala J, Koivisto H, Järvi K, Anttila P, Niemelä O. Immune responses to ethanol metabolites and cytokine profiles differentiate alcoholics with or without liver disease. Am J Gastroenterol 2005;100:1300-10.

30. Tuma DJ, Klassen LW. Immune responses to acetaldehyde-protein adducts: Role in alcoholic liver disease. Gastroenterology 1992;103:1969-73.

31. Worrall S, de Jersey J, Shanley BC, Wilce PA. Antibodies against acetaldehyde-modified epitopes: An elevated IgA response in alcoholics. Eur J Clin Invest 1991;21:90-5.

32. Green PH, Jabri B. Celiac disease. Annu Rev Med 2006;57:207-21.

33. Koivisto H, Hietala J, Anttila P, Niemelä O. Co-ocurrence of IgA antibodies against ethanol metabolites and tissue transglutaminase in alcohol consumers: Correlation with proinflammatory cytokines and markers of fibrogenesis. Dig Dis Sci 2008;53:500-5.

34. Metzger MH, Heier M, Mäki M, Bravi E, Schneider A, Löwel H, et al. Mortality excess in individuals with elevated IgA anti-transglutaminase antibodies: The KORA/MONICA Augsburg cohort study 1989-1998. Eur J Epidemiol 2006;21:359-65.

35. Powell AE, Davies HTO, Thomson RG. Using routine comparative data to assess the quality of healthcare: Understanding and avoiding common pitfalls. Qual Saf Health Care 2003;12:122-8.

36. Needham DM, Scales DC, Lapaucis A, Pronovost PJ. A systematic review of the Charlson comorbidity index using Canadian administrative databases: A perspective on risk adjustment in critical care research. J Crit Care 2005;20:12-9.

37. Ventura A, Magazzu G, Greco L. Duration of exposure to gluten and risk for autoimmune disorders in patients with celiac disease. SIGEP Study Group for Autoimmune Disorders in Celiac Disease. Gastroenterology 1999;117:297-303.

38. Ventura A, Neri E, Ughi C, Leopaldi A, Citta A, Not T. Glutendependent diabetes-related and thyroid-related autoantibodies in patients with celiac disease. J Pediatr 2000;137:263-5.

39. Chin RL, Sander HW, Brannagan TH, Green PH, Hays AP, Alaedini A, et al. Celiac neuropathy. Neurology 2003;60:1581-5.

40. Frustaci A, Cuoco L, Chimenti C, Pieroni M, Fioravanti G, Gentiloni $\mathrm{M}$, et al. Celiac disease associated with autoimmune myocarditis. Circulation 2002;105:2611-8

41. Rehm J, Roerecke M. Reduction of drinking in problem drinkers and all-cause mortality. Alcohol Alcohol 2013;48:509-13. 